\title{
Nebular diagnostics of hot star atmospheres
}

\author{
Grażyna Stasińska ${ }^{1}$, Daniel Schaerer ${ }^{2}$, and Claude J. Zeippen ${ }^{1}$ \\ ${ }^{1}$ Laboratoire de l'Univers et de ses Theories, \\ Observatoire de Paris-Meudon, F-92195 Meudon, la France \\ ${ }^{2}$ Laboratoire d'Astrophysique, Observatoire de Midi-Pyrénées, \\ 14 avenue E. Belin, F-31400 Toulouse, la France
}

\begin{abstract}
The ionization structure of nebulae depends on the spectral energy distribution of the ionizing stars. Therefore nebulae are potential tools to check the validity of the energy distribution predicted by stellar atmosphere models. However, the problem is complicated by the fact that the ionization structure also depends on the nebular density distribution and that the predictions of photo-ionization models strongly rely on atomic physics computations, which bear their own uncertainties.
\end{abstract}

\section{Introduction}

Stellar atmosphere modeling is complex and requires assumptions. Predictions shortward of the Lyman limit cannot be tested by observations of stars due to absorption of the radiation by the surrounding gas. In principle, observation of emission lines from this gas can be used to test the model atmospheres. Such tests are important, since emission line nebulae probe the massive star content (e.g., obscured $\mathrm{H}$ II regions in the Milky Way, giant $\mathrm{H}$ II regions in distant galaxies) and thus the star formation history in various parts of the Universe. Emission-line nebulae are also powerful abundance indicators in galaxies.

\section{Some recent O-type star model atmosphere codes}

Below is a non-exhaustive list of recent codes to compute stellar atmospheres:

(i) COSTAR (Schaerer \& de Koter 1997): spherical expanding atmospheres, radiative transfer with Sobolev approximation; H-He in full non-LTE, metal blanketing using opacity sampling with Monte-Carlo simulations;

(ii) CMFGEN (Hillier \& Miller 1998): spherical expanding atmospheres, radiative transfer in comoving frame using non-LTE + line-blanketing with super-level approach for metals;

(iii) WM-basic (Pauldrach et al. 2001): spherical expanding atmospheres, radiative transfer in observer's frame, detailed non-LTE for metals, line-blocking and -blanketing with ALI + opacity sampling; and

(iv) TLUSTY (Hubeny \& Lanz 1995): plane parallel atmospheres in hydrostatic and radiative equilibrium, fully blanketed non-LTE, millions of lines.

These codes predict substantially different Lyman continuum flux distributions. 


\section{Impact on nebular lines}

The $\mathrm{H} \beta$ luminosity is governed by the number of H-ionizing photons $Q\left(\mathrm{H}^{0}\right)$. $\mathrm{He}$ I $5876 / \mathrm{H} \beta$ (resp. He II $4686 / \mathrm{H} \beta$ ) are governed by - but not necessarily proportional to - the ratios $Q\left(\mathrm{He}^{0}\right) / Q\left(\mathrm{H}^{0}\right)$ (resp. $Q\left(\mathrm{He}^{+}\right) / Q\left(\mathrm{H}^{0}\right)$ ) of $\mathrm{He}^{0}$ (resp. $\mathrm{He}^{+}$) to $\mathrm{H}$-ionizing photons. The ratios of lines from two adjacent ions, e.g., [O III] $5007 /[\mathrm{O} \mathrm{II}] 3727$ depend on the hardness of the spectral energy distribution - but also on the ionization parameter $U=Q\left(\mathrm{H}^{0}\right) /\left(4 \pi R^{2} n c\right)$. The radiation softness parameter $\eta=([\mathrm{O} \mathrm{II}] /[\mathrm{O} \mathrm{III}]) /([\mathrm{S} \mathrm{II}] /[\mathrm{S} \mathrm{III}])$ (Víchez \& Pagel 1988) is much less dependent on $U$. Finally, the energy gains in a nebula are larger for a harder radiation field, resulting in a larger electron temperature, and therefore a larger [O III $] 4363 / 5007$.

\section{Tests of model stellar atmospheres using photo-ionized nebulae}

\subsection{Comparing samples of $\mathrm{H}$ II regions with model grids}

Such an approach has been adopted by Sellmaier et al. (1996), Stasińska \& Schaerer (1997), Giveon et al. (2002), and Morisset et al. (2003).

The first step is to identify diagrams where the effects of Lyman continuum flux distribution are important. For example, Figure 1 indicates that the $[\mathrm{Ar} \mathrm{III}] 8.9 \mu /[\mathrm{Ar} \mathrm{II}] 6.9 \mu$ versus $[\mathrm{Ne} \mathrm{III]}] 15.8 \mu /[\mathrm{Ne} \mathrm{II}] 12.8 \mu$ is a potentially interesting diagram.

The second step is to test the role that the density distribution of the nebula might have on the diagrams.

The third step consists in assessing the effect of atomic data uncertainties. It is generally believed that collision rates calculated recently with state-ofthe art methods and computer codes are statistically accurate to within $20 \%$. However, specific transitions can still be problematic (see, for example, the 50odd papers in the Atomic Data from the Iron Project series published in A\&A, and the references therein ${ }^{1}$. The situation is not as satisfactory for recombination coefficients and charge transfer reaction rates (see e.g., the reviews by Badnell 2001 and Stancil 2001). In particular, dielectronic recombination coefficients for elements in the third row could be inaccurate by factors of about 2 (see, e.g., Ferland et al. 1998). Indeed, due to the lack of complete experimental spectroscopic data, one must rely on purely theoretical structure calculations with inherent uncertainty on the positions of the autoionizing levels. Concerning charge exchange reactions, estimates using the Landau Zeener approximation are likely to be wrong by factors $2-10$. The consequences of such uncertainties on photo-ionization models must be addressed. A simple way to do so is to vary arbitrarily the rate coefficients between the lower and upper limits of the error bars estimated by atomic physicists. As astrophysical models and observations improve, there will be more pressure on atomic data producers to refine their accuracy estimates, as well as to reduce the inaccuracies in the data offered to specialists.

The fourth step is the confrontation with the observations. For example, using the $[\mathrm{Ar} \mathrm{III}] 8.9 \mu /[\mathrm{Ar} \mathrm{II}] 6.9 \mu$ versus $[\mathrm{Ne} \mathrm{III}] 15.8 \mu /[\mathrm{Ne} \mathrm{II}] 12.8 \mu$ diagram and

\footnotetext{
${ }^{1}$ More information can be found at http:// ww. usm.uni-muenchen.de/people/arch4/iron-project.html
} 

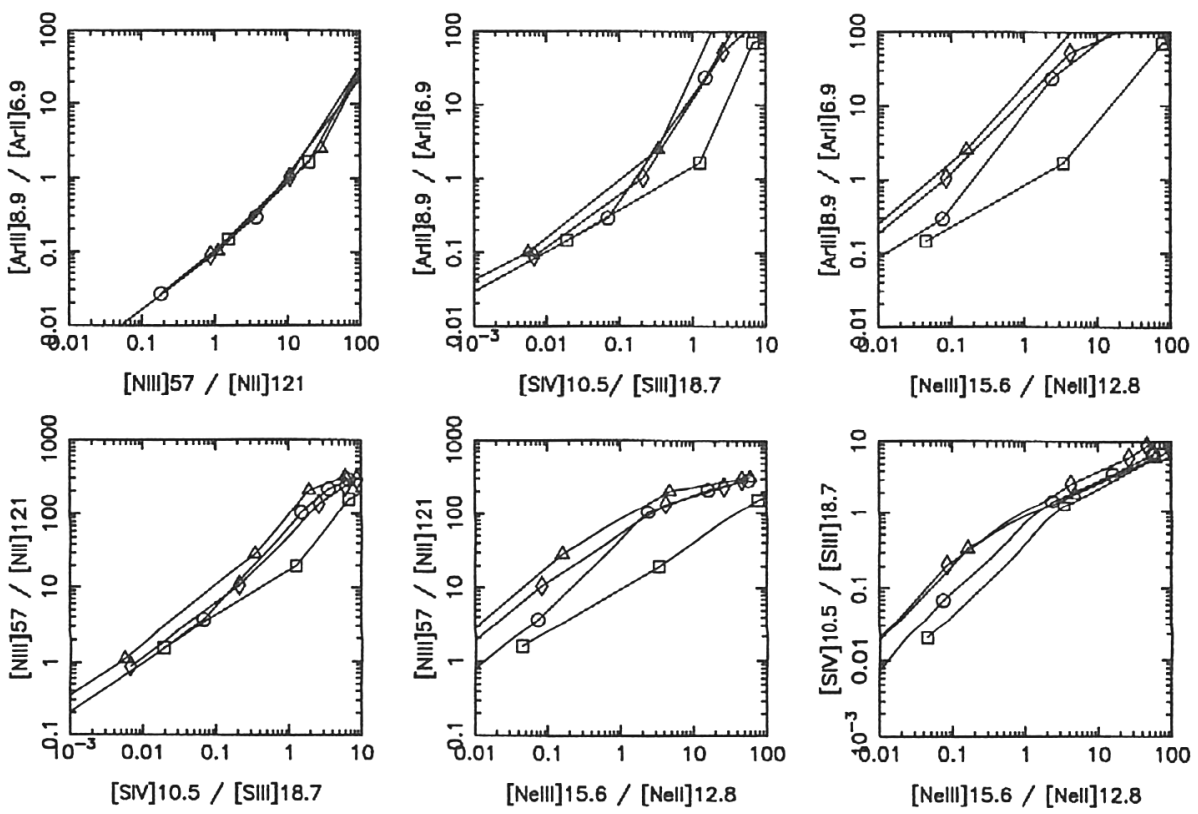

Figure 1. IR emission-line ratio diagrams for sequences of photo-ionization models corresponding to different stellar atmospheres. The photo-ionization models are computed with the code PHOTO. Models using WM-basic atmospheres are represented by circles, COSTAR by squares, CMFGEN by diamonds, and Kurucz 1991 by triangles. In each sequence, the star temperature goes from $30000 \mathrm{~K}$ to $55000 \mathrm{~K}$. The nebulae are homogeneous spheres with $n=10^{4} \mathrm{~cm}^{-3}$ excited by a single main sequence star. Solar metallicity is assumed.

confronting models with ISO observations of Galactic $\mathrm{H}$ II regions, Giveon et al. (2002) concluded that WM-basic atmospheres better explains the observations than the much softer LTE Kurucz (1991) atmospheres. As seen from Figure 2, other non-LTE line-blanketed atmospheres including stellar winds yield overall similar results. A more detailed analysis, taking into account stars of various luminosity classes as well as metallicity effects on the stars and gas, is presented in Morisset et al. (2003). Inherent problems are: $(i)$ uncertainties due to beamsize effects; (ii) uncertain de-reddening ([Ar III] affected by silicate band); (iii) possible contribution of several stars to the ionization while the models consider single star ionization; and $(i v)$ geometry of the model grid not representative of the real geometry of the H II regions.

\subsection{Detailed model fitting of individual $\mathrm{H} I I$ regions}

Such an approach has been followed by Esteban et al. (1993), Crowther et al. (1999) and Oey et al. (2000). The most thorough study so far is that of Morisset et al. (2002), who constructed a photo-ionization model of the compact H II region G 29.96-0.02, for which the exciting star is of spectral type between $\mathrm{O} 5$ and $\mathrm{O} 8$. A core-halo density structure is needed to reconcile the density derived from the $[\mathrm{O}$ III $] 52 \mu / 88 \mu$ line ratio with the r.m.s. density derived 

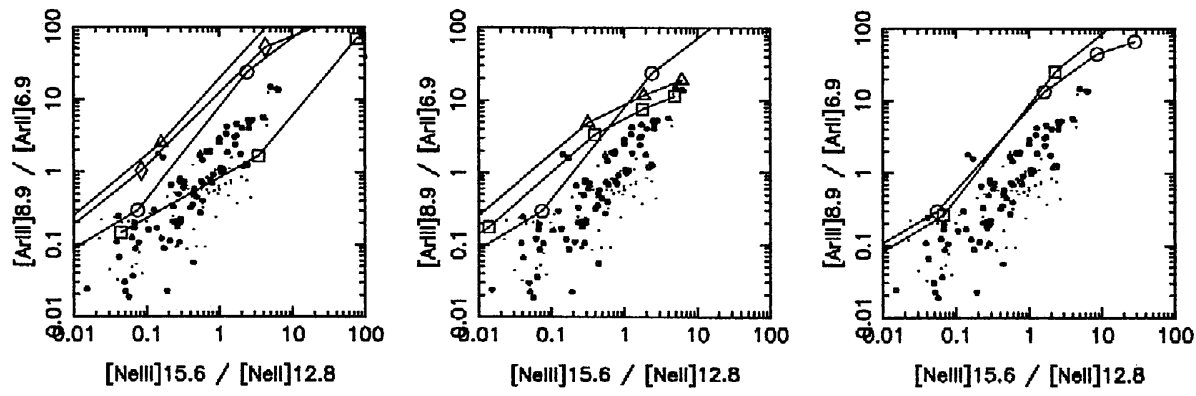

Figure 2. [Ar III] $8.9 \mu /[\mathrm{Ar} \mathrm{II}] 6.9 \mu$ vs. [Ne III] $15.8 \mu /[\mathrm{Ne} \mathrm{II}] 12.8 \mu$ models and observations, ISO data from Peeters et al. (2002) and Giveon et al. (2002). Objects where only an upper limit is available for at least one of the four intensities are represented by smaller circles. Left: the same models as in Figure 1. Middle: WM-basic models with different density structures. Circles: same models as in Figure 1. Squares: models with $n=10^{4} \mathrm{~cm}^{-3}$ and filling factor $10^{-2}$. Triangles: models with same ionization parameter but for a thin bubble geometry. Right: wM-basic models with different atomic data. Circles: same data as in Figure 1. Squares: the dielectronic recombination of $\mathrm{Ar}^{+}$has been arbitrarily increased by a factor of 5 .

from radio observations (the infrared [O III] lines are collisionally de-excited in the densest regions) and beamsize effects are fully taken into account for each individual line. The conclusion of this study is that COSTAR atmospheres provide the best fit to the 17 observational constraints. However, reasonable fits are also obtained with CMFGEN, WM-basic or TLUSTY atmospheres, adopting a higher effective temperature. Further tests of model atmospheres are obviously needed.

\section{Should one be desperate about the use of nebular spectra for astrophysical inferences?}

The answer is no. Let us illustrate this on the emission line sequence of metalpoor H II galaxies. H II galaxies are powered by clusters of coeval stars. They show significant trends between line ratios and $\mathrm{H} \beta$ equivalent width (a first order estimate of the age of the stars). Qualitatively, some of these trends can be explained by the gradual softening of the ionizing radiation field as the most massive stars disappear. However, photo-ionization models assuming homogeneous spheres and pure starbursts fail to account quantitatively for all the observed diagrams (Stasińska et al. 2001). Stasińska \& Izotov (2003) have reconsiderend the problem using a sample of $400 \mathrm{H}$ il galaxies, divided into metallicity bins. Each bin is compared with models of appropriate metallicity. The adiabatic expanding bubble model produces the adequate increase in time of $[\mathrm{OI}] 6300 / \mathrm{H} \beta$, which had found no explanation so far. An additional old stellar population is not sufficient to reproduce observed slopes in diagrams relating line ratios and $\mathrm{H} \beta$ equivalent width, calling for the necessity of invoking a covering factor decreasing with time. Such a scenario explains the observed trends irrespective of the atmosphere models, the main driver being the lifetime of the ionizing stars and the evolution of the gas. However, the models show that the observed He II 
$4686 / \mathrm{H} \beta$ cannot be accounted only by Wolf-Rayet stars and requires additional $\mathrm{X}$-rays, either produced by the present burst or by the older stellar population.

\section{Conclusion}

Testing the atmospheres of hot stars using nebular lines is feasible in principle but difficult. It is not certain that the most elaborate stellar atmosphere models today yield the correct Lyman continuum fluxes. Nevertheless, ionized nebulae remain a good tool to investigate the interaction between massive stars and interstellar matter and the chemical evolution of galaxies, provided one stays aware of the possible problems.

\section{References}

Badnel, N.R. 2001, in: G. Ferland \& D.W. Savin (eds.), Spectroscopic Challenges of Photo-ionized Plasmas, ASP-CS 247, p. 37

Crowther, P.A., Pasquali, A., de Marco, O., et al. 1999, A\&A 350, 1007

Esteban, C., Smith, L.J., Vílchez, J.M., Clegg, R.E.S. 1993, A\&A 272, 299

Ferland, G.J., Korista, K.T., Verner, D.A., et al. 1998, PASP 110, 761

Giveon, U., Sternberg, A., Lutz, D., et al. 2002, ApJ 566, 880

Hillier, D.J., Miller, D.L. 1998, ApJ 496, 407

Hubeny, I., Lanz, T. 1995, ApJ 439, 875

Kurucz, R.L., 1991, in: L. Crivellari, I. Hubeny \& D.G. Hummer (eds.), Stellar Atmospheres: Beyond Classical Models, NATO ASI Series C, Vol. 341 (Dordrecht: Kluwer), p. 441

Morisset, C., Schaerer, D., Martín-Hernández, N.L., et al. 2002, A\&A 386, 558

Morisset, C., Schaerer, D., Bouret, J.-C., Martins, F. 2003, A\&A in press

Oey, M.S., Dopita, M.A., Shields, J.C., Smith, R.C. 2000 ApJS 128, 511

Pauldrach, A.W.A., Hoffmann, T.L., Lennon, M. 2001, A\&A 375, 161

Peeters, E., Martín-Hernández, N.L., Damour, F., et al. 2002, A\&A 381, 571

Schaerer, D., de Koter, A. 1997, A\&A 322, 598

Sellmaier, F.H., Yamamoto, T., Pauldrach, A., Rubin, R.H. 1996, A\&A 305, 37

Stancil, P.C. 2001, in: G. Ferland \& D.W. Savin (eds.), Spectroscopic Challenges of Photo-ionized Plasmas, ASP-CS 247, p. 3

Stasińska, G., Schaerer, D. 1997, A\&A 322, 615

Stasińska, G., Schaerer, D., Leitherer, C. 2001, A\&A 370, 1

Stasińska, G., Izotov, Y. 2002, A\&A in press

Vílchez, J.M., Pagel, B.E.J. 1988, MNRAS 231, 257

\section{Discussion}

KUDRITZKI: I wonder, whether the conclusion for your diagnostic diagram, where you compare many objects with sequences of four model atmosphere types, is limited in significance. Which of the codes fits the observations best might depend on what you assume for the properties of the underlying stars. I suggest to perform the test suggested by you in extremely well defined situations, i.e., with a very few stars exciting the H II gas (ideally only one). The stars should have well observed optical-UV spectra with an accurate spectral analysis yielding $T_{\text {eff }} / \log g$, wind properties, etc. Then the models would make a prediction for the EUV flux, which could be tested using the 
nebula emission lines.

STASIŃSKA: Indeed, that would certainly be the best way to test stellar atmospheres. The best objects for that are ring nebulae or planetary nebulae. However, even in such cases, the answers are not necessarily as clear as one would wish.

Herrero: This is to call your attention to the poster of Sergio Simón (these Proceedings), where he presents a spectral analysis for the Trapezium Cluster stars in Orion, for which we are doing exactly what Rolf Kudritzki suggested in his comment.

CERviño: I am worried about the test of atmosphere models with evolutionary synthesis codes. The codes assume by construction an infinite number of stars, but when they are compared with observations they add contributions of fractional stars, that is not physical. May that explain some of the discrepancies between theory and observations?

STASIŃSKA: If one wants to use extragalactic $\mathrm{H}$ II regions to test model atmospheres, stochastic effects are to be considered for lines that can be attributed only to a small fraction of the stars. In the case of galactic $\mathrm{H}$ II regions, the ionization is generally dominated be a single star.

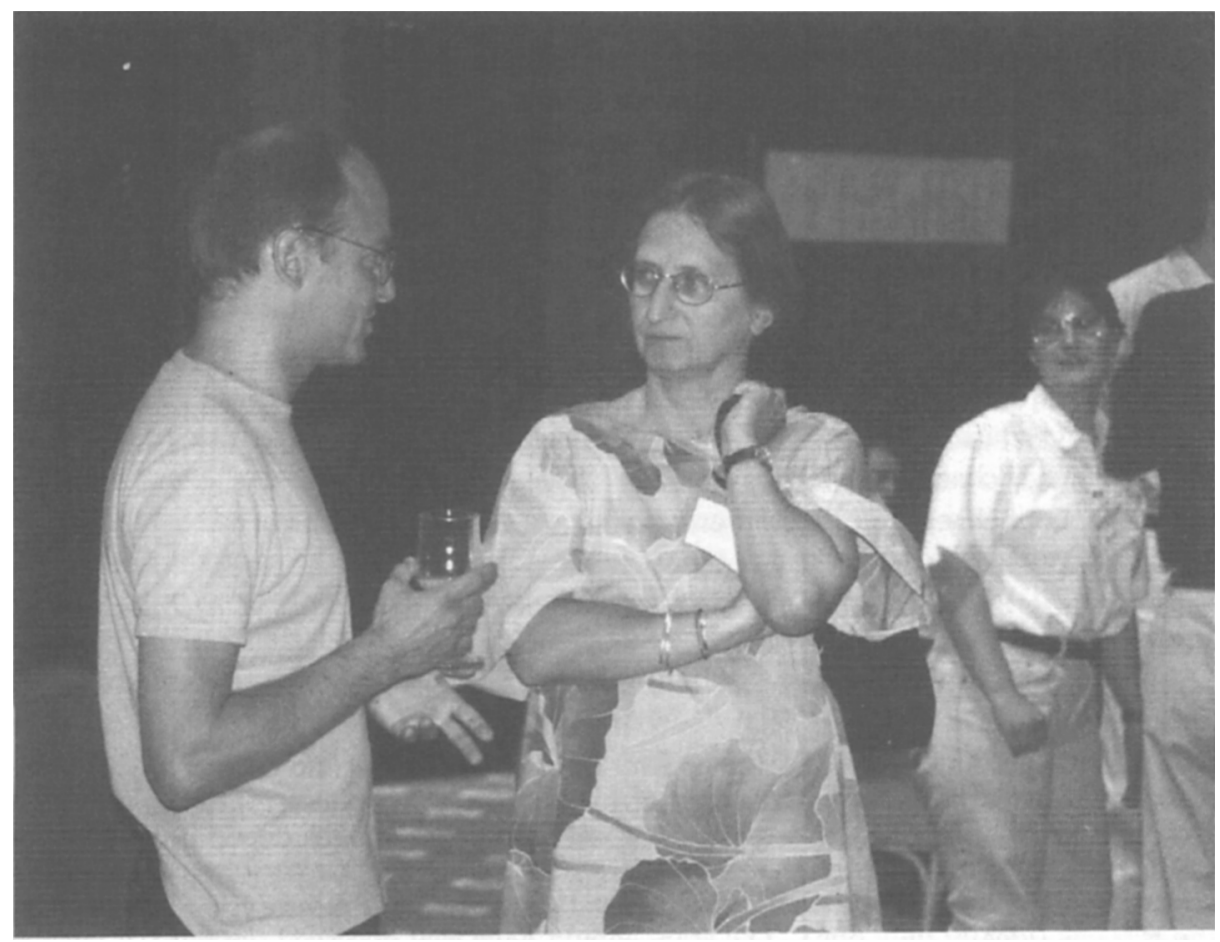

Jürgen Knödlseder and Grażyna Stasińska, continuing nebular diagnostics 